\title{
Master Manipulator with a Limitless Angular Displacement
}

\author{
Pawel Żak \\ Institute of Machine Tools and Production Engineering, Lodz University of \\ Technology, Stefanowskiego 1/15, 90-924 Lodz, Poland; pawel.zak@p.lodz.pl
}

\begin{abstract}
In the presented paper a survey of currently used popular master manipulators solutions is given, pointing their advantages and disadvantages in the process. It is pointed that all widely available devices of such type are burdened with motion limitations which affect their utilization greatly. As a solution to this problem, a novel construction of a master manipulator device is being proposed. The design process of the device itself is being discussed, but also the description of a control system is presented along with the control algorithm and filtration method (Kalman filter). The publication is being concluded with the description of experiments performed along with results obtained.
\end{abstract}

Keywords: master manipulator; construction; control system

\section{Introduction}

In the robotic field, a great number of commonly used solutions are telemanipulators [1], which main feature is that instead of replaying earlier created control program, they need to be controlled by the operator constantly to reproduce his arm motions. Such an approach is mainly to extend human capabilities, e.g. increase strength [2], provide an ability to teach the operator some typical motions by slightly guiding his hand [3], and so on. Another application of such an approach is to replace humans to ensure their safety while working in a hazardous environment, e.g. under high temperature or radiation [4], or in any type of harsh environment [5], or the combat zone [6]. The number of applications is not limited to above of course - a good example of using manipulator are medical applications. Cardio surgical robots, like daVinci [7], or its alternatives - RobIn Heart [8] or HeroSurge [9] can be pointed here. The idea behind using such solutions is to aid a surgeon during the operation - laparoscopic procedures can last for many hours, during which the personnel need to remain standing still, often in some uncomfortable position, while with robotic system help, the surgeon can sit down next to the control console, which obviously provides more comfort. Also, by using such a robot, the surgeon doesn't have to 
be sited next to the robot - there was an experiment conducted with transatlantic surgery [10], which only shows the potential of using such solutions.

To create a well-working control system that would provide full control of the operation field and would not limit the operator's awareness of the environment, it is necessary to equip the robotized system with a set of sensors to create a feedback loop to the operator, especially force feedback which "transfers" touch sense from the machine to operating hand [11]. Such a system enables the surgeon to, e.g. use the palpation examination method without using his fingers, also the facture, rigidness, etc. of the tissues can be checked by touching it with the robot [12]. Another possibility to use such a system is to slightly guide the surgeon's hand while performing some typical gestures [13], like sewing. It can be either used to control the correctness of performed procedures - on a basis of a database created during several earlier treatments, or it can serve as a simulator and training utility for students during their medical studies [14].

The last thing to be taken care of while creating a telemanipulator system is a proper construction master manipulator, also called a haptic device, which serves as a bridge between the operator and a manipulator. Such device should be characterized by high ergonomics - it should not limit operator's movements at any time, as such limitation breaks the fluency of work [15] - hand's motion is being stopped without any significant (from operator's point of view) reason after what some kind of clutch needs to be pressed to move the handle back to the center of a workspace.

There is a great number of commercially available solutions of haptic devices. Also, the problem of a limited workspace is well-known. Therefore, the manufacturers of master manipulators try to solve it using a variety of methods, yet none of them provide an ultimate solution to the problem. They tend to make the operator unable to reach such unwanted configurations of the haptic devices. There are three commonly used methods the designers use to avoid reaching the haptic device workspace range.

The first method is to create the master manipulator in form of an exoskeleton perfectly fitted to the operator's arm. The solution uses the operator's motion limitations - he is not able to move anywhere away from his reach, therefore no artificial boundaries appear during the operation.

A clear example of such a device is Able [16]. The device was created by the Haption company. The device is being offered in the market and can be used in a variety of different applications. Another example is Active A-Gear presented in [17]. This device is used for rehabilitation purposes, not to control anything, yet it possesses the features of haptic devices created under this principle. Another example a 7-dof haptic device is described in [18]. Its only purpose is to control medical robots. 
All the above examples show that besides the advantage pointed out earlier, such devices also have some disadvantages. The main disadvantage is the lack of the universality of such a solution - each device needs to be customized for the operator before it can be used to provide the assumptions given earlier in this section. Also, such a solution should not be fixed to the operator's back, as it is supposed to be light, therefore, such a system requires a lot of space and cannot be moved easily if needed.

Another possibility is to create a haptic device with a heavily extended workspace which limits are far beyond the operator's reach. Such a solution has been proposed i.e. by Haption in Scale1 device [19].

In this case, the device requires a frame with rails that supports the grip held by the operator. The fact is that he can walk freely around the room as the grip will follow him fluently, therefore, his motions are not restricted in any way.

Another interesting example is iFeel6BH1500 device wider described in [20]. In this case, the user's interface is being located in the middle of the frame and is being held by a number of cables. The cable's ends are coupled with drives fixed to the frame. The motions of the user pull some of the cables, while others are being released. The rotations of motors shafts are being calculated into interface orientation. A similar solution was introduced by Haption in INCA [21].

The fact is, that this solution solves the problem of linear limitations completely, as the operator movements are not limited in any way, yet both of the presented devices have enormous volumes, therefore the application has to be limited to some large rooms. Also, the usage of some of these devices can be hazardous to the operator, as the used motors generate a great torque that might easily break the user's arm, which is possible when adjusting the haptic device to its own applications using API. The other disadvantage is quite obvious when the size of the overall device is taken into account - fixing the device to the ground might even require some heavy equipment. It needs to be added that the problem of orientation limitations remains unsolved in these devices.

A known solution for overcoming orientation limitations in haptic devices is to add an additional degree of freedom to the kinematic chain. Such additional joint extends the maneuverability of the device, enabling the operator to move his hand without boundaries. Such a solution is being used by daVinci robot haptic device.

Such a solution solves the problem stated in previous examples of the large space required to place the device before using it. Yet, there is also a disadvantage connected to such a redundant approach - the analytical solution of such a kinematic chain becomes problematic [22] and computation complexity of the inverse kinematic problem increases [23] and the possibility of reaching the singularity of such kinematic chain remains.

Another possibility to avoid the limitations of any kind is to use an advanced vision system, for example as commercially available Kinect by Microsoft [24] in 
which the projector generates an Infrared (IR) net covering the user and the rest of the room and connected camera traces this net along with changes in its shape to detect motion. Another popular solution known from commercially available entertainment systems is the Move controller from Sony [25] where 6 DoF (Degrees of Freedom) motion of operator's hand is being detected by an IMUbased sensor system located inside a grip held by a human. Despite the fact, these solutions work well enough for entertainment purposes, they are not likely to be used as an alternative to haptic devices because it is not easy to implement force feedback features while using them.

The examples given above show that there is currently no ultimate solution to the stated problem, as mentioned at the beginning. Yet, such a solution had been found and will be presented in the following sections. It needs to be mentioned that the work will only focus on the orientation module of the haptic device, as this is the element in which singularities might appear. The device should be also as light as possible. The ideal solution would be to make the operator move his arm without feeling that he is using any kind of device at the same time. Yet, such systems do not exist currently. This article provides a solution to a haptic device that is characterized by a limitless workspace.

\section{The Device}

The development of the device free of drawbacks defined in the Introduction began with its construction design based on an analytical approach and the creation of assumptions for the control system and algorithms used. The description of these parts is divided into two parts presented in the following subsections.

\subsection{Design Assumptions}

To achieve an unlimited workspace of the manipulator, one needs to change the approach to the construction design and use an element that could move freely and without boundaries. The idea behind the proposed solution can be seen in Fig. 1.

The idea behind the proposed solution is that it consists of a sphere (1) that serves as a user interface (the operator is holding that part). Next to the sphere, there is a permanent magnet (2) that pulls the sphere towards itself. The sphere does not touch the magnet as it lies on three Omni wheels (3) that are mounted on drives (4) shafts. Omni wheels were added to the system to provide the possibility to transfer torque onto the sphere, which is required to enhance the device with a force feedback feature. The sphere is made of ARMCO 03J pure iron, which is a ferromagnetic alloy with low magnetic memory. 


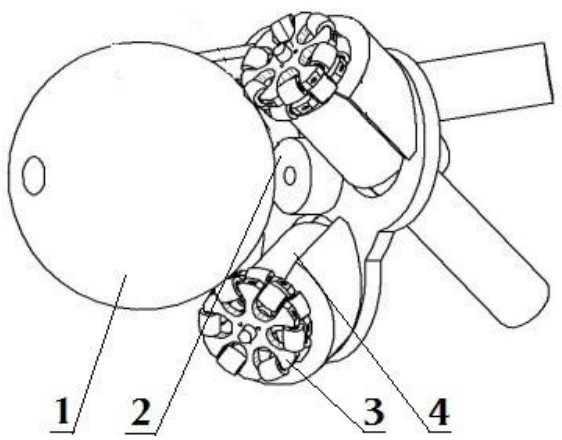

Figure 1

A concept of the device [27]

Also, the sphere is divided into two halves and is hollow. That is because of a fact that it also serves as a housing for the sensor system. The overall device needs to be oriented as shown in Fig. 1, as the working principle of the sensor system requires it [26].

The mentioned sensor system uses an IMU consisting of three triaxle sensors: an accelerometer, a magnetometer, and a gyroscope. The sensor is also equipped with a power source and a Bluetooth interface that provides communication with a control unit. The purpose of the sensor system is to determine the actual orientation of the sphere on a basis of acceleration and magnetic field vectors, while data obtained from the gyroscope serve as feedback for the filtration method. The magnetic field source provided by the magnet is much stronger than Earth's magnetic field and fields generated by the drives. As mentioned before, the magnet's second purpose is to pull the sphere next to itself resulting in its stable position. Although drives are used in the construction are equipped with encoders, they are not being used in the orientation calculation.

The orientation of the sphere is being derived by a combination of two vectors: Earth gravity and the magnetic field of the permanent magnet shown in Fig. 1. As a result of the constant orientation of the presented device, these vectors are always non-parallel, thus a rotation matrix can be defined with Eq. 1:

$$
\mathbf{R}=\left[\begin{array}{lll}
A_{\mathrm{i}} \times M_{\mathrm{i}} & A_{\mathrm{i}} \times\left(A_{\mathrm{i}} \times M_{\mathrm{i}}\right) & A_{\mathrm{i}}
\end{array}\right]
$$

where:

$A_{\mathrm{i}}$ - acceleration unit vector components,

$M_{\mathrm{i}}$ - magnetic unit vector components.

A matrix presented in the given form fully describes the actual orientation of the sensor unit and a sphere. The derivation process had been thoroughly described in [27]. Data obtained from the sensors serve not only to determine the orientation 
but also as the input for the Kalman filter developed for this solution to obtain the highest accuracy measurement possible. It needs to be pointed that matrix (1) is used only once in the orientation determination process to obtain preliminary values - after that, it is the filter's duty to estimate the orientation of the system based on mentioned input. The only disadvantage of using a rotation matrix to define the orientation of the object is calculation complexity, as in each iteration all 9 elements need to be taken into account. Therefore, it has been decided to switch to some other orientation representation method. Quaternion algebra has been selected, for orientation described that way still fully represents object's state and preserves rotation matrix main advantage - lack of singularities occurrence, while the number of generated quaternion elements is four. Also, using quaternion algebra to describe the orientation is much more convenient in the case of developing mentioned Kalman filter, for all the elements of the unit quaternion are dependent on each other. Additionally, calculation of quternion elements direvatives is well known. The transformation from matrix to quaternion has been done according to [28].

As mentioned, the user interface is being pulled toward three Omni wheels. To ensure constant one-point contact between the sphere's surface and each of the wheels, their rolling elements shape had been geometrically determined [29]. It was necessary to select a proper material pair for elements being in contact to achieve maximum friction. The material selected for the rollers is Polyamide (PA6), which is characterized by a ca. 0.45 friction coefficient against steel [30]. The created Omni wheel construction can be seen in Fig. 2.

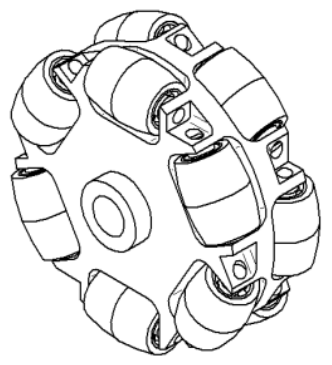

Figure 2

The isometric view of the designed Omni wheel [29]

It was necessary to determine the Jacobian matrix for the proposed device construction to achieve the possibility to control the drives on which Omni wheels are mounted. The result is the presence of a force feedback feature in the device. As the kinematic structure of the device is not typical, the standard Jacobian matrix derivation method has not been used. Instead, the derivation procedure had been based on the geometry of the device and vectors describing the relevant displacements of the drives [31], which are shown in Fig. 3. 

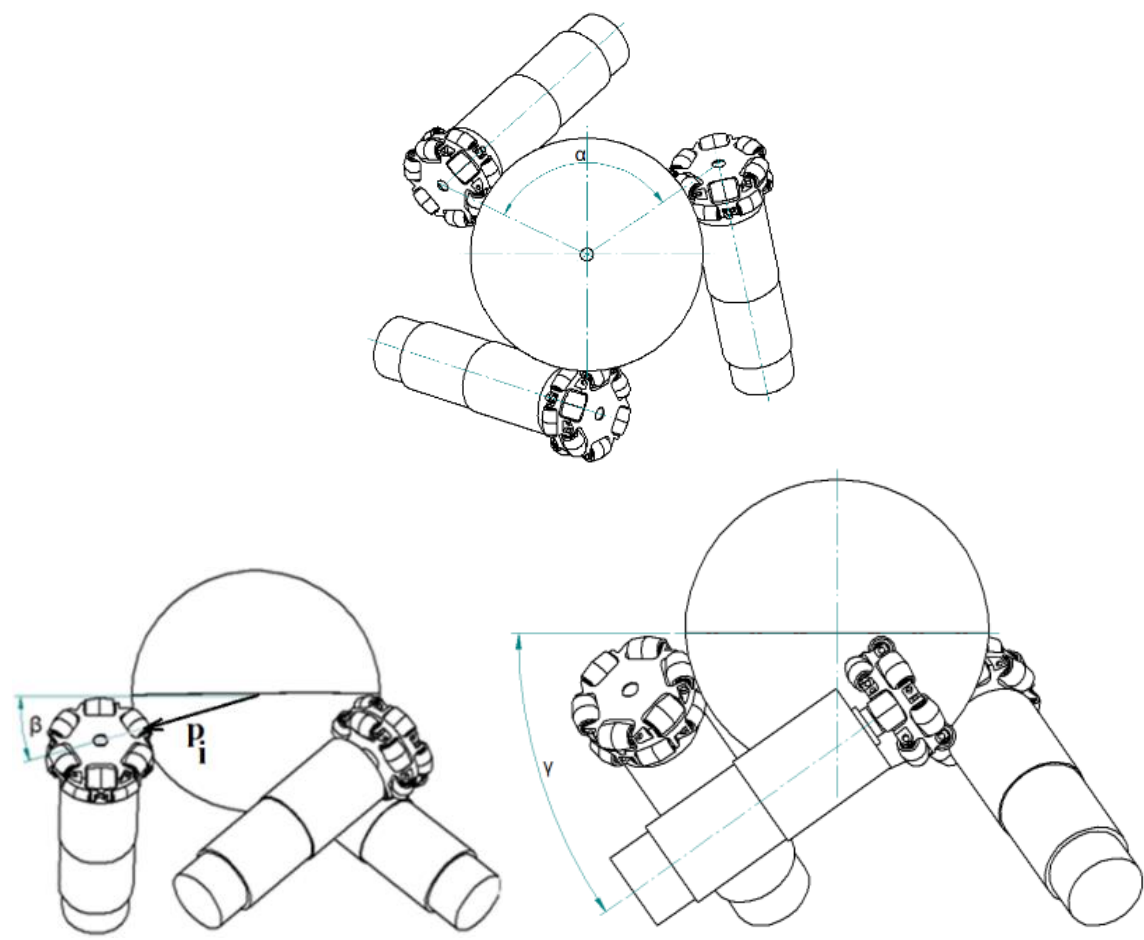

Figure 3

Geometrical dependencies of the device [31]

The outcome Jacobian matrix takes the following form:

$I=\frac{\left(f^{-1}\right)^{D}}{\operatorname{det} J^{-1}}$

Where

$J^{-1}=\frac{R}{\gamma} *\left[\begin{array}{c}\sin \alpha_{i} * \sin \gamma+\cos \alpha_{i} * \sin \beta * \cos \gamma \\ -\cos \alpha_{i} * \sin \gamma+\sin \alpha_{i} * \sin \beta * \cos \gamma \\ \cos \beta * \cos \gamma\end{array}\right]^{T}$

$\mathrm{R}$ - radius of the sphere, equal $35 \mathrm{~mm}$,

$\mathrm{r}$ - radius of the Omni wheel, equal $15 \mathrm{~mm}$,

$\alpha_{\mathrm{i}}$ - angular position of the individual drives,

and the Jacobian matrix determinant presented in equation (2) is equal

$$
\operatorname{detj}^{-1}=\mathrm{c} \beta * \mathrm{c} \gamma *\left(\mathrm{c} \beta^{2} * \mathrm{c \gamma ^{2 }}-1\right) *\left(s\left(\alpha_{1}-\alpha_{2}\right)-s\left(\alpha_{1}-\alpha_{2}\right)+s\left(\alpha_{2}-\alpha_{2}\right)\right)
$$


It is worth noticing that all the elements of equation (4) are constant. Therefore, the resulting determinant value differs from zero, which is the ultimate proof that the presented device is free of singularities. The optimum orientation of the individual drives has also been determined using a derived Jacobian matrix.

\subsection{Control System}

The overall control system loop can be seen in Fig. 4.

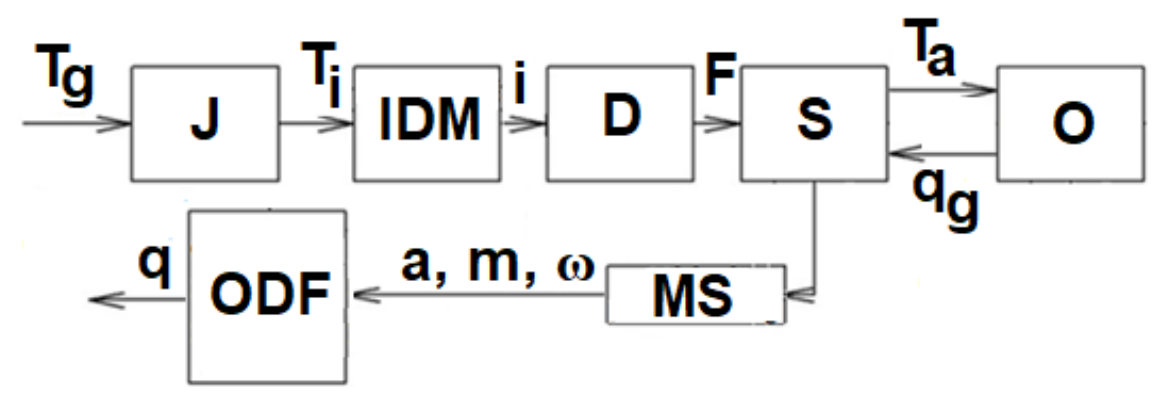

Figure 4

Haptic device control system schematic

The input for the control system is a Torque value ( $\mathrm{T}_{\mathrm{g}}-$ given torque) that should be generated according to the actual state of the manipulator controlled by the given haptic device. By using the derived Jacobian matrix $(\mathrm{J})$ the input is being calculated to input torques $\left(\mathrm{T}_{\mathrm{i}}\right)$ for the individual drives. Basing on the Inverse drive model (IDM) the control currents (i) are being calculated and passed to the drives (D). Their motion is being transferred by the friction force (F) to the sphere (S) which is being held by the operator $(\mathrm{O})$, therefore, the outcome torque $\left(\mathrm{T}_{\mathrm{a}}-\right.$ actual torque) generated in the sphere serves as an output of the system. Yet, there is another input - the operator who is in control of the device makes rotation with his hand providing the system with orientation change of the sphere (described using quaternions, $\mathrm{q}_{\mathrm{g}}$ - given quaternion). A measurement system (MS) located inside the sphere detects changes in the orientation by measuring acceleration, magnetic flux, and angular velocity vectors $(\mathrm{a}, \mathrm{m}, \omega)$. Based on their values, an algorithm determines the actual orientation of the object and performs the filtration process (ODF - Orientation determination and filtration), providing corrected data on a measured angular displacement of the sphere (q).

The mentioned filtration method is being based on Extended Kalman Filter (EKF) [32]. To make use of the filter possible in this case, it is necessary to correctly select elements of the state vector that describe the actual state of the measured object. It was decided that this vector should be:

$$
x=\left[\begin{array}{lll}
q_{\mathrm{i}} & \omega_{\mathrm{i}} & b_{\mathrm{i}}
\end{array}\right]^{T}
$$


where

$q_{i}$ - quaternion elements in which $\mathrm{q}_{\mathrm{o}}$ is scalar element,

$\omega_{i}-$ angular velocity elements,

$b_{i}-$ gyroscope's bias elements.

Mentioned bias elements are taken into account in the case of this filter, a sensor on which the measurement unit is being based is a low-cost model and it was assumed that measured values will change over time. A measurements vector of EKF is being constructed with values that are being measured in the process, which is:

$y=\left[\begin{array}{lll}a_{m i} & m_{m i} & \omega_{m i}\end{array}\right]^{T}$

where:

$a_{m i}-$ measured acceleration vector elements,

$m_{m i}-$ measured magnetic vector elements,

$\omega_{m i}-$ measured angular velocity vector elements.

As EKF is a recurrent filter type, it requires an object's state in the next time step to be defined. For a given state vector, such prediction takes the following form:

$x_{\mathrm{t}+1}=\left[\begin{array}{c}q_{\mathrm{i}}+\Delta q_{\mathrm{i}} \\ \omega_{\mathrm{i}}+\Delta \omega_{\mathrm{i}} \\ b_{\mathrm{i}}\end{array}\right]$

where:

$\Delta q=\dot{q} \cdot \Delta t$

Quaternion derivative from equation (8) can be calculated according to [33] and

$\Delta \omega_{i}=\varepsilon_{i}$

where $\varepsilon_{i}$ is an angular acceleration value in a given direction. Its value needs to be determined during the filter creation process. In the given case, the standard acceleration value corresponds to the operator's hand motions and for the filter's creation purpose it was defined as equal $2 \mathrm{~m} / \mathrm{s}^{2}$, for exceeding this value seems unlikely in the typical haptic device application.

The last three elements of the state vector (7) describe the bias of the gyroscope. Besides noise data acquired with the used gyroscope is also burdened with bias the initial values were taken by reading the angular velocity of the fixed device. The reading should be equal, yet they are not and it was assumed that this difference is to change its volume over time. Bias elements were added into the state vector and included in equations describing angular velocity to estimate bias variations and to remove its impact on the readings. 
Further creation of the EKF for the given example does not deviate from the standard EKF procedure and the details and overall process description can be found in [34].

\section{Experiments}

The experimental phase of the created device was divided into two parts. The first part is connected to define mechanical parameters of the device, such as the maximum transferable force and repeatability of force generation by the force feedback feature. Another part was the tests done on created measurement unit to determine its features, like filtration correctness, repeatability of measurement, resolution of the sensor system.

\subsection{Torque Transfer Level}

One of the assumptions of the described project was to use materials pair providing maximum friction to obtain drive transmission in 3 Degrees of Freedom. As mentioned in Section 1.1, the assumption was that sphere is made of some kind of metal, while the Omni wheels rollers material is polyamide. It became necessary to check what is the maximum transferable torque for the final assembly of created elements and selected materials. To perform this task, an experiment had been conducted. During the process, a 6D force sensor was used. The device had been equipped with a rode which was being pushed toward the force sensor's surface and pressed with an additional external load to make it possible to further press onto the force sensor and release the pressure in the same experiment. The test started with zero torque applied by the drives of the device it was the zero point of the measurement system. Next, a maximum negative torque of $-0.11 \mathrm{Nm}$ was applied and afterward, it was being slightly increased step-by-step until the positive maximum of $0.11 \mathrm{Nm}$ was reached. After that, the process was reversed and repeated until the maximum negative value was reached again. The step value was $0.01 \mathrm{Nm}$. The experiment was repeated 5 times for each direction of the internal device coordinate system. The result of one data set can be seen in Fig. 5 .

The maximum set values named earlier were specified during a preliminary experiment which was to detect a force for which the frictional connection between the sphere and Omni wheels becomes unstable and slippage starts to occur. It was done using the same test stand, yet the procedure was different. 


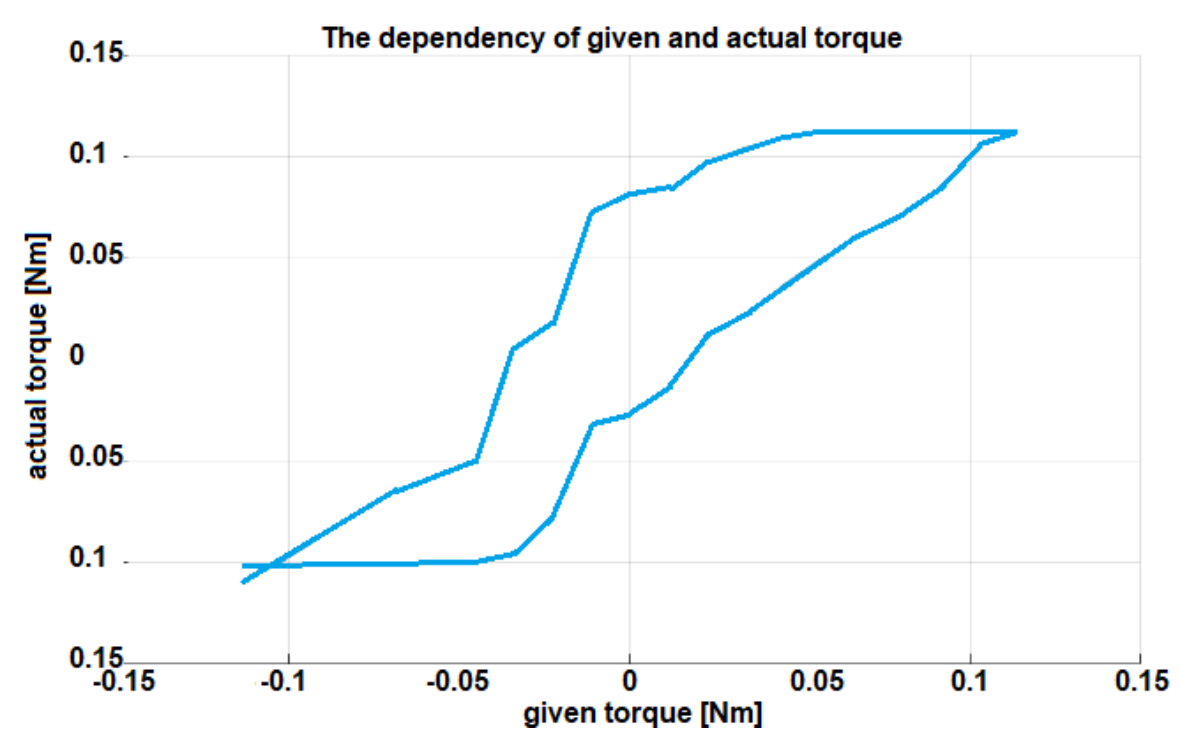

Figure 5

A dependency of set and obtained torque of the user's interface

The torques of the drives were being increased by a small step starting from 0 value, while the outcome torque of the sphere was measured. The torques of the drives were being increased until a slippage occurred. The experiment has shown that the maximum "safe" torque value is $0.11 \mathrm{Nm}$.

The actual experiment has shown that there are little losses of the torque during the process and measured values correspond to set values in boundary positions. Yet, during the motion of the device, this dependency is not so strict - as expected, hysteresis can be seen. The main purpose of this phenomenon is the presence of the backlash in cooperating elements, especially in Omni wheels rollers mounting system. The knowledge of this fact is important as it is possible to create the correction algorithm that would take into account the sureness level of torque value transfer at a set level to control the given torque value.

\subsection{Filtration Corectness}

During this experiment, the developed EKF has undergone series of tests to check the correctness of the filtration process. During all of the tests, the sensor unit mentioned in Section 1.1 was being placed inside the user's interface and set to record the data. After the acquisition process, the resulting file was processed. The motion performed by the sphere was done by the operator's hand in given sequences but also in random direction with variating velocity dependent on operator selection. The sampling rate was $100 \mathrm{~Hz}$. 
In Fig. 6 a scalar and two imaginary elements of the quaternion are presented. Raw data from the sensor unit was taken to calculate the quaternion value to compare it with the corresponding value estimated by the Kalman filter. This calculation based on raw data is made only for these tests and is not being used anywhere in the algorithm.

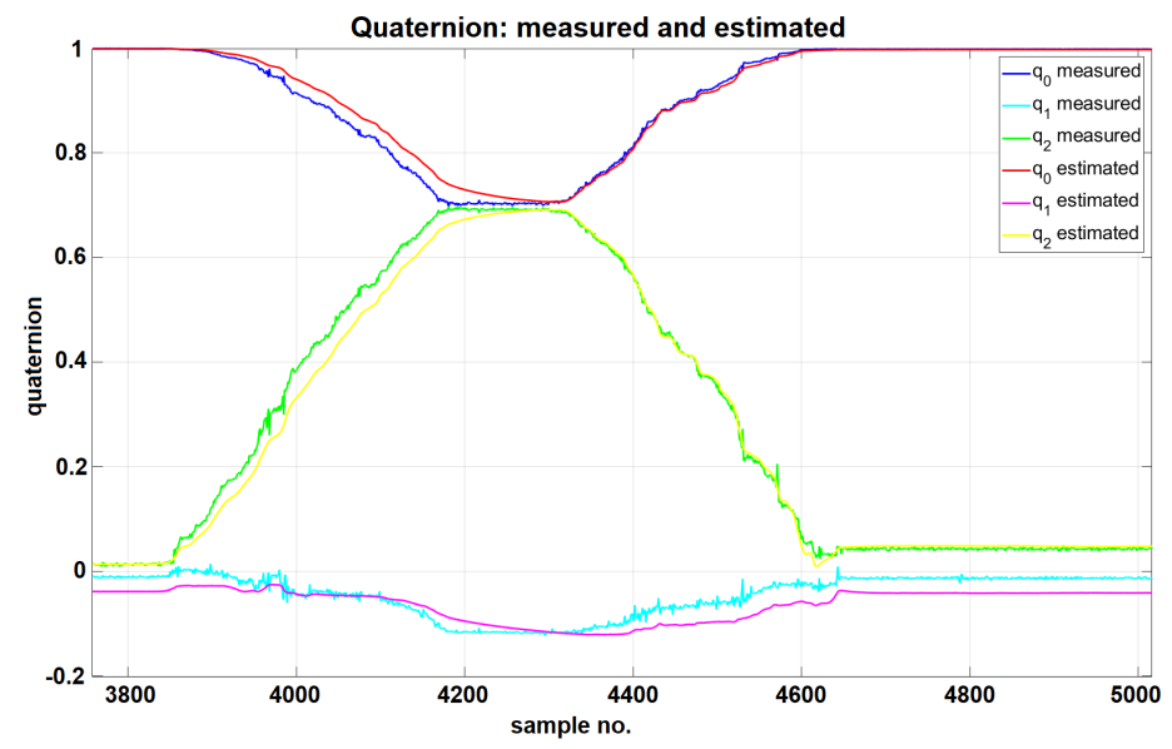

Figure 6

Quaternion filtration

It can be seen that in the stationary phases (e.g. samples $0-1500$ ) actual and estimated values are slightly moved from each other. This is an outcome of the fact that estimation based on the data collected from three different sensors and their fusion and is acceptable.

The estimated quaternion values presented in Fig. 6 are based on filtered data obtained from sensors specified earlier. The estimation results of this collection can be seen in Fig. 7, an accelerometer in this case. It needs to be mentioned that during the filtration procedure, only raw data from the sensor are taken into account. Filtered readings are only used to check filtration process correctness.

As it can be seen, the phenomena present in the previous figure, are present in accelerometer estimation as well. The estimated value follows the actual one perfectly, yet corresponding values are slightly moved next to each other. The origin of the displacement has already been analyzed. 


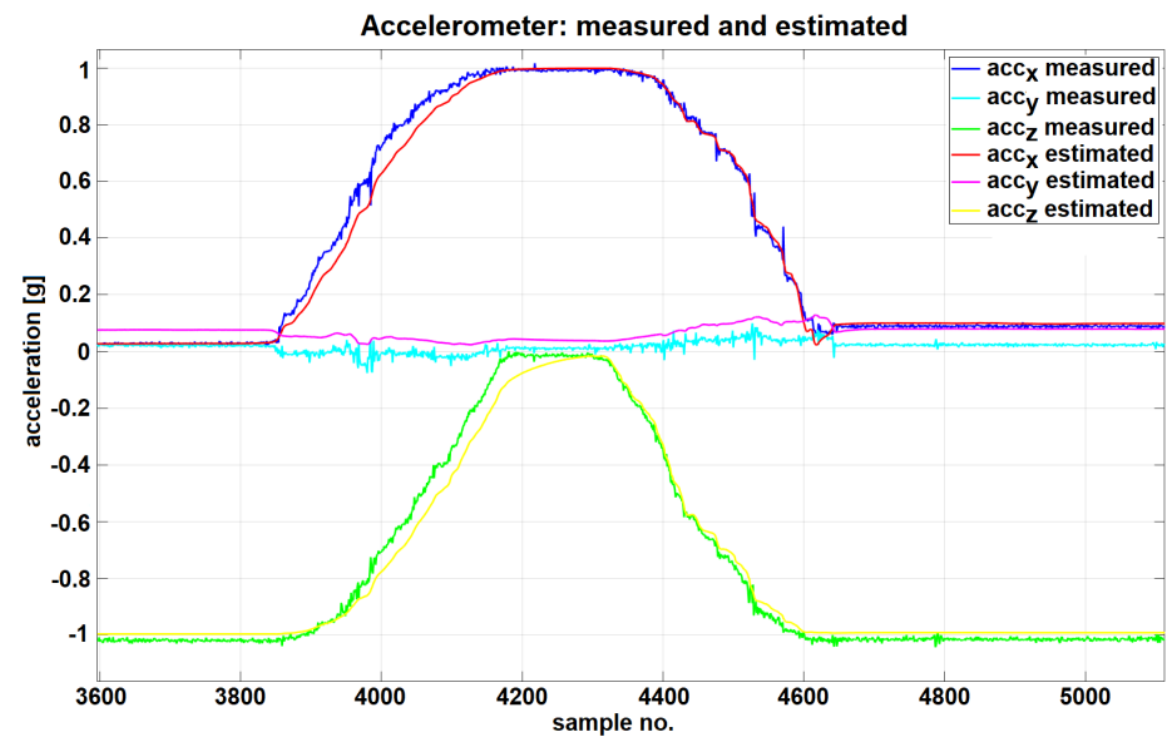

Figure 7

Accelerometer filtration

\subsection{Repeatability}

In the repeatability of the measurement test, the haptic device had been equipped with a measurement rode which could be pressed against a prismatic base. It resulted in the stable and repeatable orientation of the interface when pressed. During the experiment the rode was being moved away from the prism and pressed again towards it - the process has been repeated 30 times. Each time the rode was being pressed, its orientation was being stabilized and measured using a built-in sensor system. The angle values of the position to be obtained were determined by moving the rode to the prism for the first time, and after waiting a couple of seconds to make self-vibrations of the system damp, the reading has been made.

The point cloud obtained during the experiment can be seen in Fig. 8. 


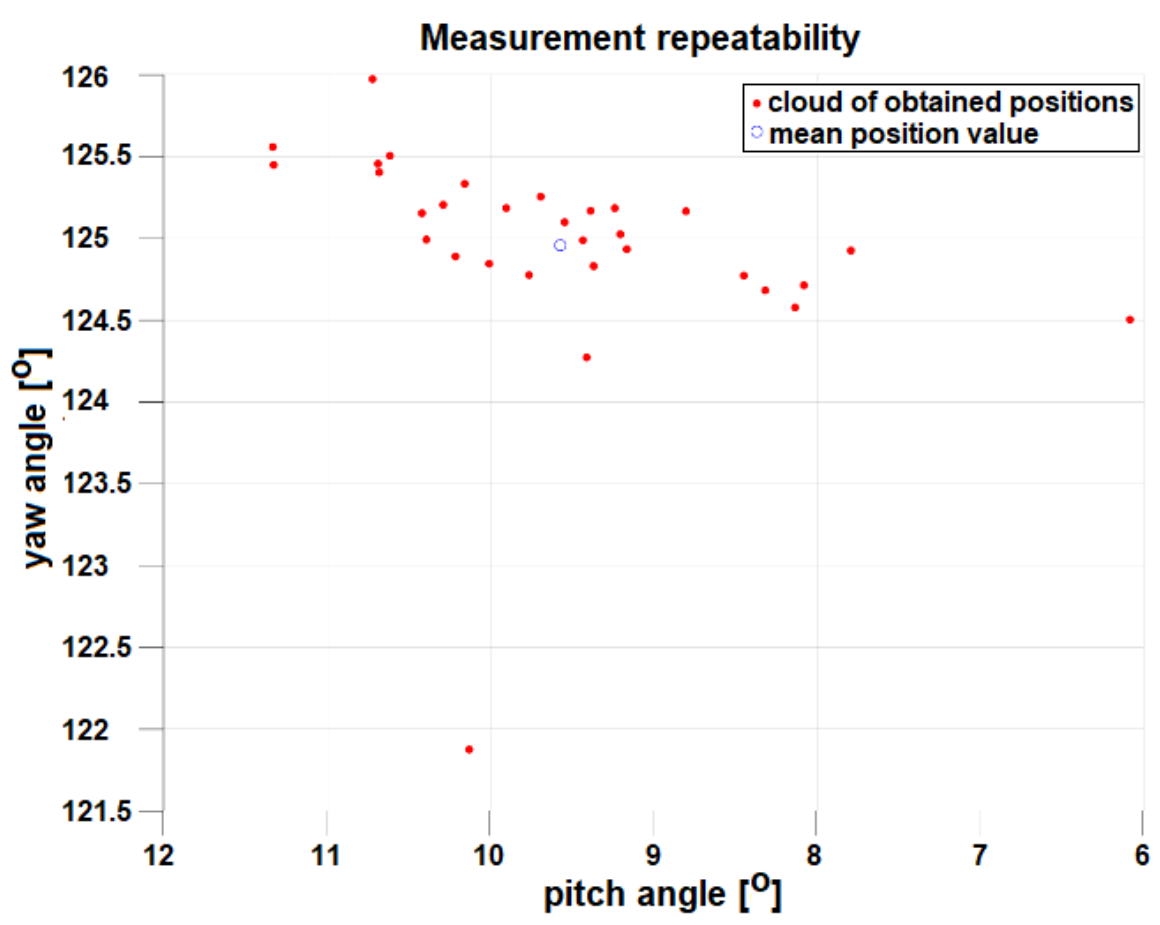

Figure 8

Measured positions in subsequent approaches to the set orientation presented in pitch-yaw plane

The blue point visible in the Figure represents the mean value of all obtained points in the cloud. The obtained point cloud can be used to determine the value of the repeatability parameter with the use of the equation provided in [35]:

$P_{\alpha}=\frac{\sum_{j=1}^{\mathrm{u}} \sqrt{\left(\overline{R l}-R \mathrm{I}_{j}\right)^{2}+\left(\overline{P C h}-P C h_{j}\right)^{2}+\left(\overline{\mathrm{Y}}-Y_{j}\right)^{2}}}{n}$

where

$\overline{R l}, \overline{P c h}, \bar{Y}-$ mean value of the obtained angle,

$R l_{j}, P c h_{j}, Y_{j}-$ actual values of obtained angles.

Obtained repeatability for the described measurement system was calculated as $P_{\alpha}=1.18^{\circ}$.

The creators of commercially available master manipulators seldom share this parameter with the audience, yet it can be found that in the case of Cybergrasp haptic glove it is ca. $1^{\circ}$ [36], which shows that the obtained result proves the possible applicability of the created device in this matter. 


\subsection{Resolution}

To specify the minimum detectable displacement that can be sensed by the sensor system, a test on the resolution has been performed. To conduct this experiment, a test stand has been constructed. The idea behind this test was to make the sphere containing a sensor system (1) rotate by a given value. To ensure the maximum precision and repeatability of subsequent rotations, the stand has been equipped with a Maxon Motor servo drive (2). It can be seen in Fig. 9.

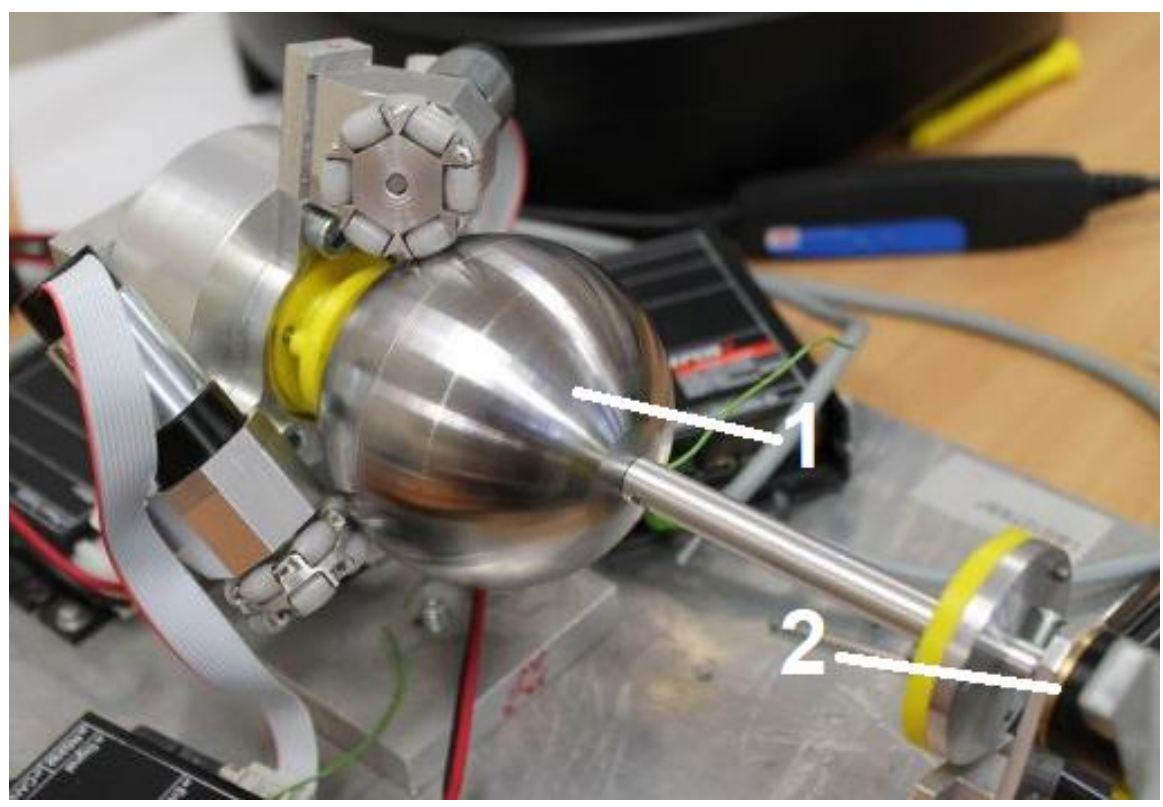

Figure 9

Test stand for resolution measurement

During the test, a set of rotations was done with the drive's shaft resulting in the user's interface and sensor system rotation at the same time. The rotation angle value was being changed between the sets and results read by the sensor were recorded. The purpose was to find displacement value for which it wouldn't be possible to distinguish individual steps. The displacement value range starts with $1^{\circ}$ and ends with $0.006^{\circ}$, which was the minimum value possible to obtain by used servo drive. 


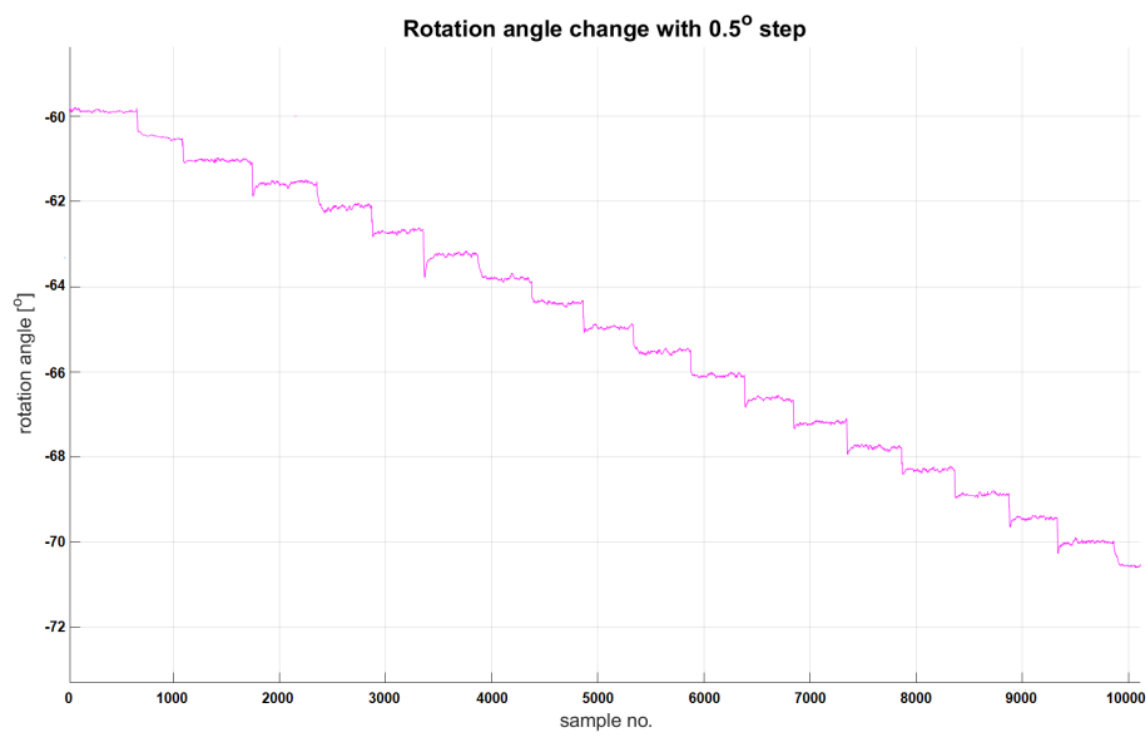

Figure 9

Diagrams showing a set of rotations with $0.5 \mathrm{dg}$ step

For the motions with the displacement, steps equal $1^{\circ}$ and $0.5^{\circ}$ (Fig. 9) the obtained value was big enough to allow a clear reading of a rotation angle. It also showed that measured rotation angle corresponds to a given one, which additionally proves that the developed fusion data algorithm and orientation determination procedure is effective and works correctly.

The rotation angle step has been further decreased to $0.1^{\circ}$ value. The sensor system was able to detect such displacements, yet the increment value is lower than the present noise of the reading, therefore the obtained angular value can be only shown with some approximation.

\section{Discussion}

As the performed experiment results have shown, the proposed device can be used to control manipulators (their orientation) while providing force feedback and quite precise orientation determination while giving no artificial restrictions to the operator's hand motion. Yet, there is still room for improvements, for example, a correction algorithm should be added to the control system which was schematically presented in Fig. 4. It is planned to add correction value to the $T_{i}$ element to avoid torque losses resulting from backlash shown in Fig. 5. To fully determine the sureness level of torque transfer it is necessary to perform additional 
experiments in a dynamic setup in which the device would be in motion during force application.

Additional works are planned to be done on the filtration algorithm - it is planned to add additional filtration used during really slow and precise motions to increase resolution value over the values shown in Fig. 9. Also, the dynamics of the created filtration method will be checked to determine how the system reacts to fast and rapidly changing motions of the sphere. Also, the completed control system (with mentioned correction algorithm loop) will be fully described, including its transmittance calculation.

The planned works will be summarized by the creation of a new measurement unit that will be equipped with all the features shown in the paper and listed above, as for now, its only purpose is to collect orientation data from the sensors - as was stated earlier.

\section{Conclusions}

This article presented a thorough description of a novel master manipulator device, in which the main feature is a limitless orientation motion and the fact that it is being free of singularities occurrence. The survey of existing commercially available, as well as experimental haptic devices has been done to point advantages and disadvantages of such mechanisms. It showed that all of them try to avoid singularities occurrence by making the user unable to reach such a position, without trying to solve the problem completely. This work presented the creation process of the device that is free of named issues. The idea behind the construction has been explained, the design process has been described, there is also the description of the Jacobian matrix necessary to implement the force feedback feature to assist the operator during his work. Also, a control system of the device was presented and thoroughly described, taking into account the explanation of constructed measurement unit, its work principle, and an algorithm used to calculate the orientation of the connected object. Also, the description of the used filtration method has been presented, along with the explanation of basic assumptions needed to be done during the filter creation process.

The constructed device had undergone the experimentation process to check its applicability, main features, characteristics. The maximum force possible to be transferred by the friction in a given case has been defined to check the volume of force feedback felt by the operator. The accuracy and repeatability of the measurement unit have been determined during two experiments. Also, the correctness of created data filter was tested and verified. All the experiments, test stations, and results were presented and discussed. The overall experimentation process showed that created device is working according to the assumptions and can be used in professional applications. 


\section{References}

[1] V. Ciobanu, N. Popescu, A. Petrescu, Robot telemanipulation system, IEEE conference on System Theory Control and Computing (ICSTCC), Vol. 17, pp. 681-686, October 2013

[2] A. Barrow, W. Harwin, Design and Analysis of a Haptic Device Design for Large and Fast Movements, Machines, 4(1), 2016

[3] M. Boroujeni and M. Misagh. Daly: Haptic Device Application in Persian Calligraphy, Proceedings of the 2009 International Conference on Computer and Automation Engineering, 2009

[4] J. Kutuvan: Remote Handling Technology in Nuclear Industry, Modern Manufactuing India, Vol. 1, Issue 3, 2013

[5] S. Sivčev, J. Coleman, E. Omerdić, G. Dooly, D. Toal: Underwater manipulators: A review, Ocean Engineering, Vol. 163, pp. 431-450, 2018

[6] E. Amareswar, G. S. S. K. Goud, K. R. Maheshwari, E. Akhil, S. Aashraya and T. Naveen: Multi purpose military service robot, 2017 International conference of Electronics, Communication and Aerospace Technology (ICECA), Coimbatore, 2017, pp. 684-686, doi: 10.1109/ICECA.2017.8212752

[7] J. Leven et al.: DaVinci Canvas: A Telerobotic Surgical System with Integrated, Robot-Assisted, Laparoscopic Ultrasound Capability, Lecture Notes in Computer Science (Vol. 3749), pp. 811-818, 2005

[8] L. Podsędkowski, P. Żak: Tests on cardiosurgical robot robin heart 3, Robot Motion and Control (Vol. 396), pp. 433-442, 2009

[9] M. Moradi Dalvand, S. Nahavandi, M. Fielding, J. Mullins, Z. Najdovski and R. D. Howe: Modular Instrument for a Haptically-Enabled Robotic Surgical System (HeroSurg), IEEE Access, Vol. 6, pp. 31974-31982, 2018, doi: 10.1109/ACCESS.2018.2844563

[10] Kent, H Nelson: Hands across the ocean for world's first trans-Atlantic surgery, CMAJ : Canadian Medical Association journal = journal de l'Association medicale canadienne 16510 (2001): 1374

[11] M. Fontana, E. Ruffaldi, F. Salasedo, M. Bergamasco: On the Integration of Tactile and Force Feedback, Haptics Rendering and Applications, 2012

[12] Batteau L. M., Liu A., Maintz J. B. A., Bhasin Y., Bowyer M. W: A Study on the Perception of Haptics in Surgical Simulation, Medical Simulation, Vol. 3078, 2004

[13] L. Podsedkowski, J. Moll, M. Moll, L. Fracczak: Are the surgeon's movements repeatable? An analysis of the feasibility and expediency of implementing support procedures guiding the surgical tools and increasing motion accuracy during the performance of stereotypical movements by the 
surgeon, Kardiochirurgia i Torakochirurgia Polska, Volume 11, Issue 1, 2014, pp. 90-101

[14] Fracczak L, Szaniewski M, Podsedkowski L.: Share control of surgery robot master manipulator guiding tool along the standard path. Int J Med Robot Jun;15(3):e1984, 2019, doi: 10.1002/rcs.1984

[15] Mugge W, Kuling IA, Brenner E, Smeets JBJ.: Haptic guidance needs to be intuitive not just informative to improve human motor accuracy, PLoS ONE, 2016, doi: 10.1371/journal.pone.0150912

[16] P. Garrec, J. P. Friconneau, Y. Measson, and Y. Perrot: Able, an innovative transparent exoskeleton for the upper-limb, Intelligent Robots and Systems, 2008

[17] P. N. Kooren, J. Lobo-Prat, M. M. H.P. Janssen, A. Q. L. Keemink, A. H. A. Stienen, I. J. M. de Groot, M. I. Paalman, R. Verdaasdonk, B. F. J. M. Koopman: Design and control of the active a-gear: a wearable 5 dof arm exoskeleton for people with duchenne muscular dystrophy, IEEE International Conference on Biomedical Robotics and Biomechatronics, 2016

[18] G. Tholey, J. P. Desai: A General-Purpose 7 DOF Haptic Device: Applications Toward Robot-Assisted Surgery, Transactions on Mechatronics (Vol. 12, Issue 6), pp. 662-669, 2007

[19] Q. Parent, J. Perret: Usability of a large-scale force-feedback device in different immersive environments, EuroVR Conference 2016, Athens, 2016

[20] Z. Chen, Y. Zhang, D. Wang, C. Li and Y. Zhang: iFeel6-BH1500: A large-scale 6-DOF haptic device, 2012 IEEE International Conference on Virtual Environments Human-Computer Interfaces and Measurement Systems (VECIMS) Proceedings, Tianjin, 2012, pp. 121-125, doi: 10.1109/VECIMS.2012.6273226

[21] J. Perret, L. Dominjon: The INCA 6D: a Commercial Stringed Haptic System Suitable for Industrial Applications, Joint Virtual Reality Conference, 2009

[22] C. Yu, M. Jin, H. Liu: An Analythical Solution for Inverse Kinematic of 7DOF Redundant Manipulators with Offset-Wrist, IEEE International Conference on Mechatronic and Automation Proceedings, pp. 92-97, 2012

[23] J. Wang, Y. Li, X. Zhao: Inverse Kinematics and Control of a 7-DOF Redundant Manipulator Based on the Closed-Loop Algorithm, International Journal of Advanced Robotic Systems (Vol. 7, Issue 4), pp. 1-12, 2010

[24] M. S. H. Abdullah, A. Zabidi, I. M. Yassin and H. A. Hassan: Analysis of microsoft kinect depth perception for distance detection of vehicles, IEEE 6th Control and System Graduate Research Colloquium (ICSGRC), Shah Alam, 2015, pp. 116-119, doi: 10.1109/ICSGRC.2015.7412476 
[25] M. Paleari, R. Luciani and P. Ariano: Towards NIRS-based hand movement recognition, 2017 International Conference on Rehabilitation Robotics (ICORR), London, 2017, pp. 1506-1511, doi: 10.1109/ICORR.2017.800946

[26] L. Podsędkowski P. Żak: Moduł zadawania położenia kątowego zadajników położenia, P.397176, 29.01.2016

[27] P. Żak: Using 9-axis Sensor for Precise Cardiosurgical Robot Master Angular Position Determination, Solid State Phenomena Vol. 199, pp 356361,2013

[28] YB. Jia: Quaternions and Rotations, Com S 477/577 Notes, Sep 10, 2013

[29] P. Żak: Ballbots rolling elements shape determination, $201621^{\text {st }}$ International Conference on Methods and Models in Automation and Robotics (MMAR), Miedzyzdroje, 2016, pp. 1143-1147, doi: 10.1109/MMAR.2016.7575299

[30] Voyer J, Klien S, Velkavrh I, Ausserer F, Diem A. Static and Dynamic Friction of Pure and Friction-Modified PA6 Polymers in Contact with Steel Surfaces: Influence of Surface Roughness and Environmental Conditions. Lubricants. 2019; 7(2):17, https://doi.org/10.3390/lubricants7020017

[31] P. Żak: Jacobian matrix determination in a novel master manipulator device, $201617^{\text {th }}$ International Conference on Mechatronics - Mechatronika (ME), Prague, 2016, pp. 1-5

[32] L. Kleeman: Understanding and applying Kalman filtering, Proceedings of the Second Workshop on Perceptive Systems Curtin University of Technology, 1996

[33] D. Xu, D. P. Mandic: The theory of quaternion matrix derivatives, IEEE Trans. Signal Process., Vol. 63, No. 6, pp. 1543-1556, 2015

[34] P. Żak: Master Manipulator Orientation Determination Method Using Extended Kalman Filter, $201818^{\text {th }}$ International Conference on Mechatronics - Mechatronika (ME), Brno, 2018, pp. 276-280

[35] EN ISO 9283:1998 standard

[36] M. Aiple and A. Schiele, "Pushing the limits of the CyberGrasp" ${ }^{\text {TM }}$ for haptic rendering," 2013 IEEE International Conference on Robotics and Automation, 2013, pp. 3541-3546, doi: 10.1109/ICRA.2013.6631073 